\title{
Development of a subsystem for automatic protection of submersible pumps based on mathematical modeling
}

\author{
Alexander V. Pilipenko*, Andrei A. Tashev, Nail K. Sharifov \\ Orel State University, Orel, Russia
}

\begin{abstract}
In this paper, the authors produce a mathematical modelling of a piston pump, develop algorithms for the operation of a protection system, taking into account the results of mathematical modelling. The authors test the mathematical model on the operation of real equipment and analyze its accuracy.
\end{abstract}

\section{Introduction}

The object of investigation in this work is a submersible vibrating pump with a constant (uncontrolled) supply of liquid. According to its design, this pump belongs to single-plunger pumps of simple acting [1,2]. This means that the fluid is pumped when the plunger moves in only one direction, and is sucked in the opposite direction. The drawbacks of singleplunger pumps include an overestimated pulsation of liquid at the outlet, which adversely affects the operation of fluid flow sensors [3]. However, the nature of the pulsation of the liquid flow at the pump outlet provides an opportunity to evaluate the pump's operation and its technical condition. In this connection, the authors made an attempt to develop an automated control system for submersible pumps with a decrease in the influence of pulsation on measuring processes and a subsystem of protection against internal technical malfunctions, which is based on the analysis of pulsation in comparison with the mathematical model [4].

The circuit of the pump and the design of the test stand for the control of the submersible pump is shown in Figure 1. Through hole I, water is drawn from a well, which enters the working cavity of the pump. Hole II serves as a branch pipe through which the water in the working cavity is transported through a conduit to a fillable tank, which in this case acts as a reservoir. Piston IV as a result of reciprocating movements creates a pressure difference, resulting in the absorption of the liquid and its movement into the water hose. Element III conditionally denotes the camera body, where all processes occur. Stem V is driven by an eclectic drive, which is powered by a $220 \mathrm{~V}$ network.

\footnotetext{
* Corresponding author: a@pilipenko.info
} 


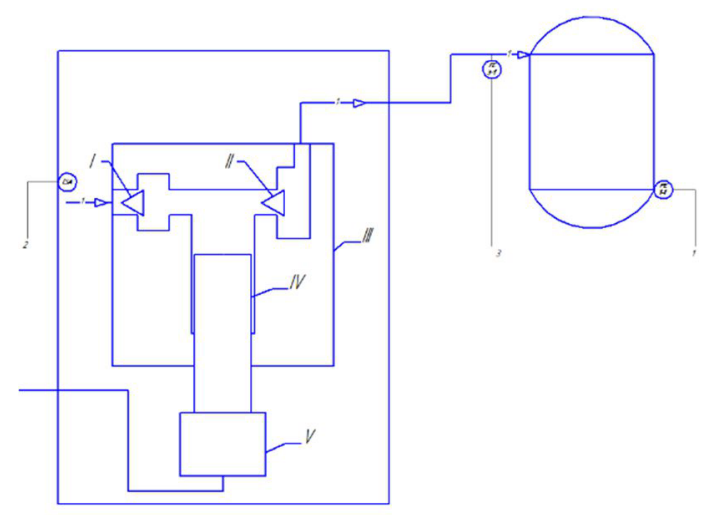

Fig. 1. Conditional scheme of the control system design.

\section{Mathematical model}

The average theoretical fluid supply of a simple-action pump can be found by the following formula (1):

$$
Q=\frac{f \times S \times n \times z}{60}
$$

where $f$ is a cross-sectional area of the plunger; $S$ is a plunger stroke; $n$ is a number of plunger strokes per minute; $z$ is a number of plungers.

We find the area of the plunger, expressing it from formula (1), with the following values of the parameters: $Q=416.67 \mathrm{~cm}^{3} / \mathrm{s} ; S=0.5 \mathrm{~cm} ; n=100 ; z=1 ; \mathrm{cm}^{2}$. These parameters were chosen according to the technical characteristics of the pump Rucheyok (Brook)

Then the plunger diameter is found by formula (2), see:

$$
D=\sqrt{\frac{4 \times 8.33}{\pi}}=3.26
$$

A characteristic feature of vibrating-type pumps is the unevenness of the liquid supply during one period. In order to realize the mathematical model of the pump's steady-state operation, a complex replacement circuit, obtained by using the mechanical analogy of a crank-plunger pump, is applied.

An instantaneous fluid delivery is determined as follows (3):

$$
Q_{\text {inst }}=f \times V \text {, }
$$

where $V$ is the velocity of the slider of the crank-slider mechanism.

We express the velocity $V$ in terms of the angular velocity of the crank shaft with a known dependence, and substitute it into the final formula (4):

$$
Q_{\text {inst }}=f \times \omega \times r \times\left(\sin \alpha+\frac{\lambda}{2} \times \sin 2 \alpha\right),
$$

where $\omega$ is angular velocity of the crank shaft; $r$ is a crank radius, $\alpha$ is an angle of crank rotation; $\lambda$ is a connecting rod ratio.

The average fluid supply of a single-plunger pump per cycle can be found by formula (5): 


$$
Q_{a v}=0.318 \times Q_{\max },
$$

where $Q_{\max }$ is a maximum instantaneous fluid delivery per cycle.

We find the angular velocity in rad/s according to formula (6) and the radius of the crank in $\mathrm{cm}$ according to formula (7):

$$
\begin{gathered}
\omega=2 \times \pi \times n=2 \times \pi \times 100=628.32 \\
r=\frac{S}{2}=\frac{5 \times 10^{-1}}{2}=0.25
\end{gathered}
$$

To find $Q_{\max }$ and construct a pump flow diagram, the MathCAD computer simulation package was applied. The result of the calculations is shown in Figure 2.

$$
\begin{aligned}
& \left.\mathrm{Q}(\mathrm{x}):=\mid \begin{array}{ll}
0 & \text { if } \sin (\mathrm{x})<0 \\
\mathrm{fp} \cdot \mathrm{w} \cdot \mathrm{r} \cdot\left(\sin (\mathrm{x})+\frac{\lambda}{2} \cdot \sin (2 \mathrm{x})\right)
\end{array}\right) \text { otherwise } \\
& \mathrm{F}(\mathrm{y}):=\mathrm{fp} \cdot \mathrm{w} \cdot \mathrm{r} \cdot\left(\sin (\mathrm{y})+\frac{\lambda}{2} \cdot \sin (2 \mathrm{y})\right) \\
& \mathrm{y}:=1 \\
& \text { Maximize }(\mathrm{F}, \mathrm{y})=1.492 \\
& \max :=\mathrm{fp} \cdot \mathrm{w} \cdot \mathrm{r} \cdot\left(\sin (1.492)+\frac{\lambda}{2} \cdot \sin (2 \cdot 1.492)\right)=1.313 \times 10^{3} \\
& \mathrm{Qsr}:=0.318 \cdot \max =417.586
\end{aligned}
$$

Fig. 2. The result of calculations in the MathCAD program

The function $Q(x)$ according to formula (4) expresses the dependence of the pump output flow on the displacement $x$, represented in radians. Since there is no flow during the reverse performance, an additional condition is introduced for plotting the graph, which excludes the reverse stroke of the crank.

The function $F(y)$ is used to find the maximum point of the function (4). To do this, the built-in Maximize function is used to find the maximum function. The following is set in it:

- function $F(y)$, where the search is performed;

- argument $y$, by which the maximization is performed.

Based on the value found, the maximum water supply max is calculated. Then the average fluid flow is calculated according to formula (5).

The graph of the instantaneous pump flow, depending on the displacement of the $Q(x)$ plunger and the average calculated liquid supply per cycle $Q s r$ is constructed in the MathCAD environment, and is shown in Fig. 3.

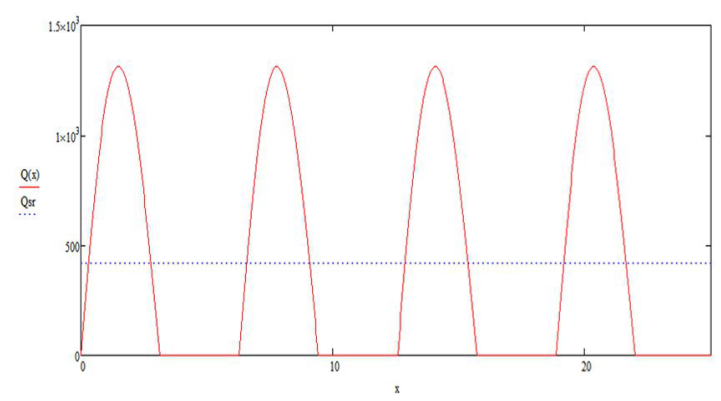

Fig. 3. The result of supply modeling 
In order to assess the adequacy of the model, let us compare the found calculated value $Q s r=417.59 \mathrm{~cm}^{3} / \mathrm{s}$ with the pump value $Q=416.67 \mathrm{~cm}^{3} / \mathrm{s}$ and estimate the accuracy of the mathematical model: $416.67 / 417.59 * 100 \%=99.7 \%$. As we can see, the accuracy of the model is high. Proceeding from this, it can be concluded that the constructed mathematical model is adequate.

\section{Algorithmization}

To protect the pump motor in the general algorithm of the automated system, the control logic was integrated, which took into account and analyzed the nature of the water supply based on the flow sensor. The developed algorithm of the pump protection subsystem is presented in the form of a block diagram in Fig. 4.

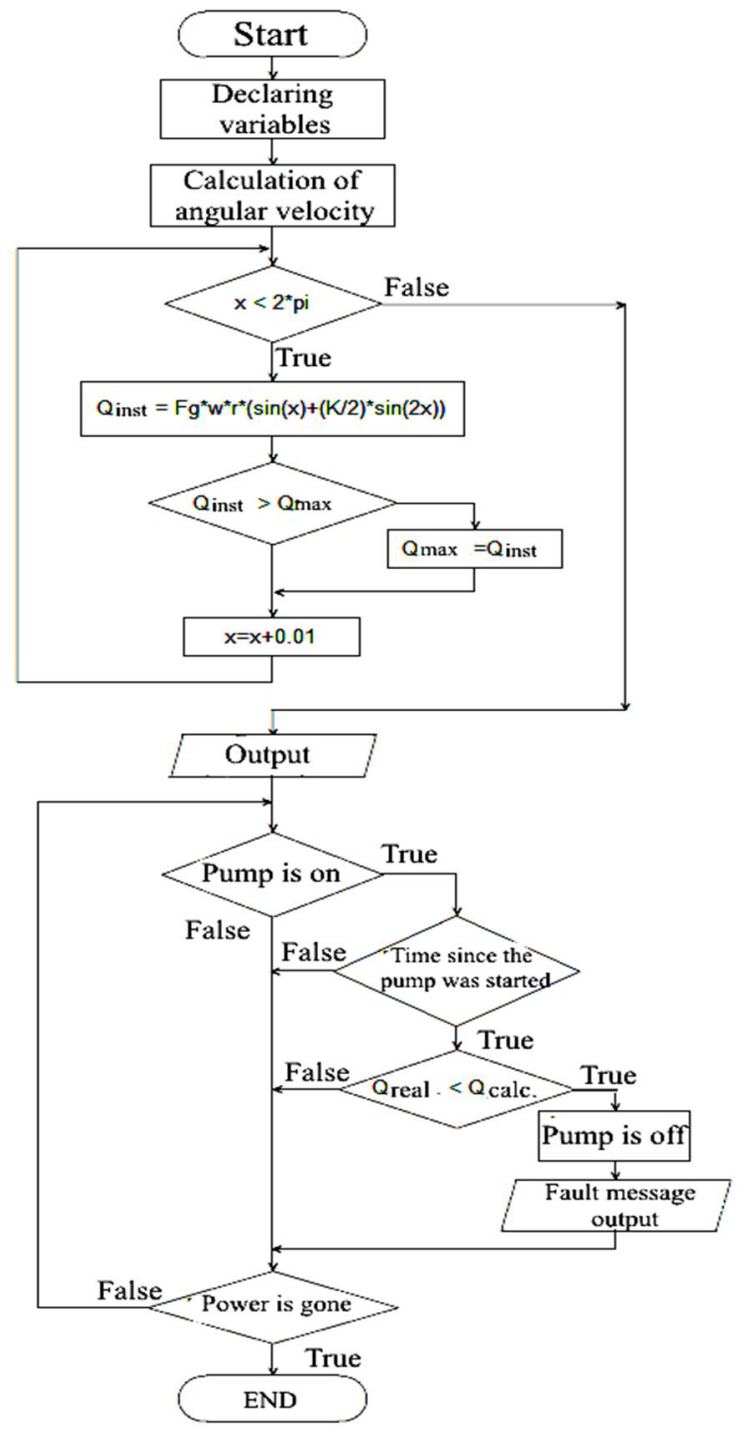

Fig. 4. Pump protecting algorithm based on a mathematical model 
First, all the variables required for the calculations are declared. Then, the mean value of the water discharge is calculated according to the code presented above. When the pump is turned on, a certain amount of time is needed for the pump to go into operation. After the start time has passed, the monitoring of the water discharge and its comparison with the calculated value begins. If the sensor readings differ from the calculated ones (considering the assumptions), then the pump turns off and sends a corresponding message to the operator.

The developed algorithm and mathematical model were tested on a test stand, the scheme of which is shown in Figure 5. Based on formula (5), the mean water discharge was calculated, which will be compared with the flow sensor reading. Since on the test stand where the system was tested and adjusted, the pump is located at a shallow depth and the length of the pipeline from the pump to the sensor is small, then the loss of pressure can be neglected and it can be assumed that the pressure on the sensor is close to the pressure at the pump outlet.

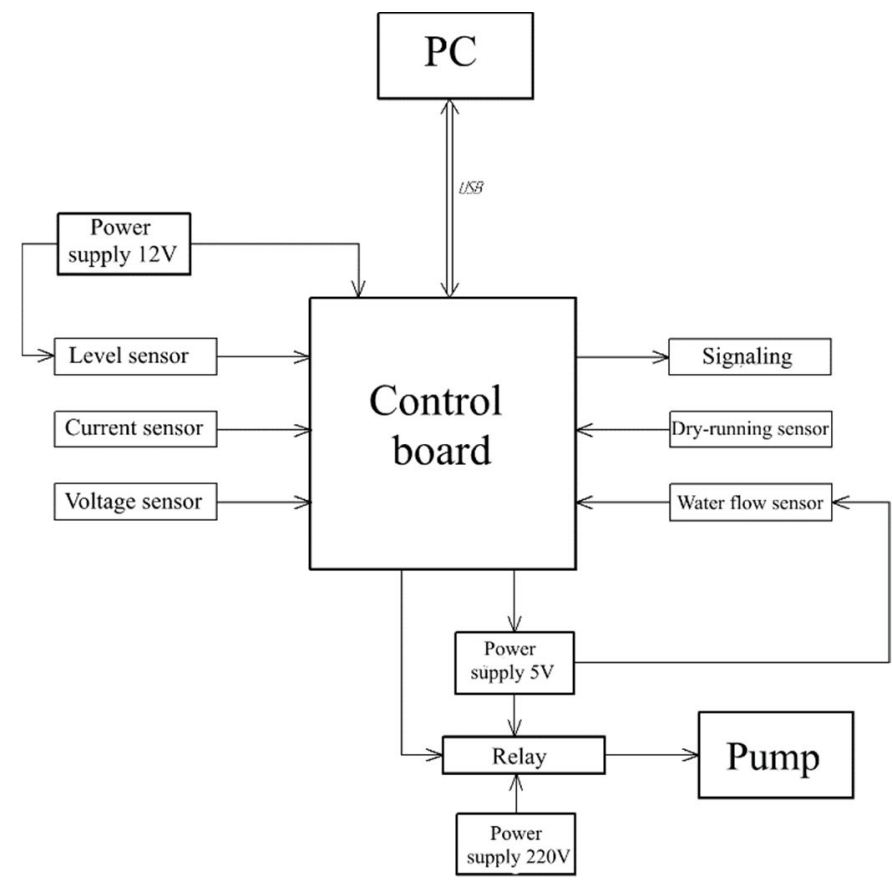

Fig. 5. Structure scheme of the test stand.

Before calculating the mean value of the fluid flow, it is necessary to specify the parameters, which are needed for the calculation, using the directive \#define:

\#define S 0.5

\#define N 100

\#define F 8.333

\#define $\mathrm{K} \quad 0.08$

Here $S$ is the stroke of the piston, expressed in centimeters; $N$ is the number of strokes of the piston per second; $F$ is the area of the piston, expressed in square centimeters; $K$ is the coefficient of the crank mechanism.

We declare the variables that will store the calculation parameters, which are needed for constructing a mathematical model:

float w;

float $\mathrm{r}$; 
Here $w$ is the angular velocity expressed in radians per second; $r$ is the radius of the crank, expressed in centimeters. These variables have a type with a floating point float, which ensures high accuracy of the calculated data.

We define variables with a zero initial value that will store the parameters used in the calculation:

float $Q$ max $=0$;

float $Q$ _inst $=0$;

float Q_mean $=0$;

float $\mathrm{x}=0$;

Here $Q \_$max is the maximum found instantaneous value of water discharge, expressed in cubic centimeters per second; $Q_{-}$inst is the instantaneous value of water discharge, expressed in cubic centimeters per second; $Q_{-}$mean is the mean found value of the water discharge, expressed in cubic centimeters per second; $x$ is displacement of the piston, expressed in radians.

First, we calculate the variables $w$ and $r$ in $\mathrm{C}$ :

$\mathrm{W}=2 * \mathrm{PI} * \mathrm{~N}$;

$\mathrm{r}=\mathrm{S} / 2$;

Here $P I$ is a constant value that is numerically equal to the number $\pi$ to within the thirtyfirst decimal place accuracy.

Using the while () cycle, we search for the maximum instantaneous water discharge using formula (4):

while $(\mathrm{x}<2 * \mathrm{PI})$

\{

$\mathrm{Q}$ inst $=\mathrm{F}^{*} \mathrm{~W} * \mathrm{r} *(\sin (\mathrm{x})+(\mathrm{K} / 2) * \sin (2 * \mathrm{x}))$;

if $\left(\mathrm{Q} \_\right.$inst $>$Q_max) $Q \_$max $=$Q_inst;

$\mathrm{x}+=0.01$;

\}

The change in displacement is carried out with a step of 0.01 , which is the result of the increment of the variable $\mathrm{x}$.

The displacement is changed in increments of 0.01 , which occurs as a result of incrementing the variable $\mathrm{x}$. Based on this variable, the instantaneous value of water discharge $Q$ inst is calculated. Then comes a logical comparison of the calculated value with the maximum instantaneous value found at a given time. If $Q_{-}$inst is greater than $Q \_$max , the new maximum value is assigned to $Q_{-} \max$. In the calculation, a range of $2 \pi$ was taken, which corresponds to the full stroke of the piston. Thus, when this value is reached, the cycle is exited.

To calculate the mean value of the water discharge $Q \_$mean, we use formula (5) and implement it in the language $\mathrm{C}$ :

Q_mean $=0.318 *$ Q_max;

As a result, we get the calculated mean of water discharge, which should be during normal operation of the pump, taking into account the assumptions specified earlier.

\section{Conclusions}

This submersible pump control subsystem was assembled and tested with simulated real pump operating conditions. All functions of pump control and protection were checked. The characteristics of the accuracy of the system were obtained and analyzed. The analysis of the obtained mathematical model was also carried out and its accuracy was estimated in the course of the system operation. The accuracy of the system is $96.8 \%$, the accuracy of the mathematical model is $99.7 \%$. Also, according to economic indicators, this system will reduce discharges for the automation of water supply to private homes by about half. 
When the mathematical model was integrated into the program code, two tasks were realized:

- the mean water discharge was calculated on the basis of the piston stroke model;

- the algorithm for protecting the pump was developed in case of a deviation from normal operation.

Based on this work, it is possible to identify the prerequisites for the following improvements:

- to realize the regulation of the pump operation with the help of a frequency converter taking into account the mathematical model for the purpose of reducing component wear;

- to install additional sensors (for example, a temperature sensor) for obtaining additional data on the pump condition;

- to implement a protocol for data exchange between the device and the remote control panel for the purpose of more flexible system setup and increase of information about the pump condition;

- to organize a database to account for the flow rate of consumed water.

\section{References}

1. K.I. Zuyev, Automation of water supply and sewage systems (Publishing house VLU Vladimir, 2016)

2. N.V. Petrov, Hydraulic presses: lecture notes: at 2 hours (Mechanical Engineering, Chelyabinsk, 1978)

3. N.V. Petrov, Dynamic processes in hydraulic presses in separating operations: monograph (Orel STU, Eagle, 2008)

4. V.P. Tarasik, Mathematical modeling of technical systems (Design of the PRO, Minsk, 1997) 